\title{
Erratum to: Morphological and Linear Scale Spaces for Fiber Enhancement in DW-MRI
}

\author{
Remco Duits • Tom Dela Haije • Eric Creusen • \\ Arpan Ghosh
}

Published online: 9 May 2013

(C) The Author(s) 2013

\section{Erratum to: J Math Imaging Vis}

DOI 10.1007/s10851-012-0387-2

In the type-setting of this article, an unfortunate error has occurred. Within this article the captions of Fig. 22 (experiment on human dataset) and Fig. 23 (experiment on artificial dataset) were accidently interchanged.

The online version of the original article can be found under doi:10.1007/s10851-012-0387-2.

R. Duits $(\varangle) \cdot$ E. Creusen · A. Ghosh

Department of Mathematics and Computer Science, Eindhoven

University of Technology, IST/e, Den Dolech 2,

Eindhoven $5600 \mathrm{MB}$, The Netherlands

e-mail: R.Duits@tue.nl

E. Creusen

e-mail: E.J.Creusen@tue.nl

A. Ghosh

e-mail: A.Ghosh@tue.nl

R. Duits · T. Dela Haije

Department of Biomedical Engineering, Eindhoven University

of Technology, IST/e, Den Dolech 2, Eindhoven 5600 MB,

The Netherlands

T. Dela Haije

e-mail: T.C.J.Dela.Haije@ student.tue.nl 